\title{
Relación entre la búsqueda de sensaciones y conductas de riesgo en adolescentes ecuatorianos desde una perspectiva de género.
}

\section{Relationship between sensation-seeking and risk behaviours in Ecuadorian adolescents from a gender perspective.}

Fecha de recepción: 15-02-2019

Fecha de aceptación: 06-06-2019

\author{
Dalila Eslava Pérez \\ Universidad Internacional de Valencia (España) \\ Ginesa López-Crespo \\ Grupo ERA. Universidad de Zaragoza (España) \\ Enrique Espinoza Freire \\ Universidad Técnica de Machala (Ecuador)
}

\section{resumen/alsstract:}

El objetivo del estudio era conocer la relación entre las diferentes dimensiones de la variable de personalidad de búsqueda de sensaciones y algunas conductas de riesgo, concretamente el consumo de drogas y la conducta sexual de riesgo, en función del género de los participantes. Asimismo, se estudió si los consumos de las diferentes drogas y la conducta sexual de riesgo estaban relacionados entre sí. La muestra contó con 367 participantes de Ecuador (un $55.6 \%$ de chicos y un $44.4 \%$ de chicas) de entre 13 y 17 años. Se administraron algunos ítems de la escala The Adolescent Risk-Taking Questionnaire y la escala Sensation-Seeking Scale-V. Los resultados mostraron que la búsqueda de experiencias y la desinhibición son las dos dimensiones que mejor explican las conductas de consumo en ambos géneros y que estos explican la conducta sexual de riesgo en chicos pero no en chicas. Además, se encontró que el consumo de drogas y las conductas sexuales de riesgo presentaron relación en ambos casos. Se plantea así la necesidad de buscar alternativas para que aquellos jóvenes con más necesidad de experimentación canalicen esa característica hacia actividades saludables.

The aim of the study was to know the relationship between the different dimensions of the sensation-seeking personality variable and some risk behaviours, specifically drug use and risky sexual behaviour, depending on the gender of the participants. It was also studied whether the consumption of the different drugs and the sexual risk behaviour were related to each other. The sample included 367 participants from Ecuador (55.6\% boys and $44.4 \%$ girls) aged between 13 and 17. Some items from The Adolescent Risk-Taking Questionnaire scale and the Sensation-Seeking Scale-V scale were administered. The results showed that the search for experiences and disinhibition are the two dimensions that best explain consumer behaviour in both genders and that these explain risky sexual behaviour in boys but not in girls. In addition, it was found that drug use and sexual risk behaviors were related in both cases. This raises the need to seek alternatives so that those young people with the greatest need for experimentation channel this characteristic towards healthy activities.

\section{palabras clave/keywords:}

Búsqueda de sensaciones, consumo de alcohol, consumo de tabaco, borracheras, conducta sexual de riesgo. Sensation-seeking personality, alcohol use, tobacco use, drunkenness, risky sexual behaviour. 


\section{Introducción}

La adolescencia es un periodo entre la niñez y la edad adulta que se inicia sobre los 10 años y termina a los 19. Se caracteriza por cambios biológicos, psicológicos y sociales, cambios generadores de crisis, conflictos y contradicciones. Los jóvenes no sólo tienen que adaptarse a los cambios corporales, si no a un nuevo contexto social (Pineda y Aliño, 2002). Además, estos contextos suelen estar cargados de emocionalidad, como lo pueden ser las situaciones sociales. Estos, entre otros, favorecen que haya una mayor frecuencia de conductas de riesgo en este colectivo (Matthys, Vandershuren y Schutter, 2013).

La Organización Mundial de la Salud (1998) define las conductas de riesgo como "forma específica de conducta de la cual se conoce su relación con una susceptibilidad incrementada para una enfermedad específica o para un estado de salud deficiente". Algunos ejemplos de estas son el consumo de drogas, la conducción temeraria, las conductas sexuales de riesgo y el comportamiento antisocial. Además, estas conductas proveen recompensas moderadas a corto plazo, lo cual las hacen atractivas, pero también proveen perjuicios, aunque estos a largo plazo (Toro-Trallero, 2010).

Paradójicamente, hay un mayor número de conductas de riesgo en la adolescencia respecto a la etapa infantil, aunque pudiese esperarse lo contrario debido a la mayor madurez del adolescente. El estudio de Oliva (2007) proporcionó información sobre los cambios cerebrales que tiene lugar en la adolescencia gracias a la resonancia magnética. Estos cambios explicarían por qué en este periodo hay más probabilidad de que se realicen conductas de riesgo, así como la existencia de esa necesidad de vivir nuevas experiencias. Por un lado, la corteza prefrontal, la encargada de la función ejecutiva y de la autorregulación de la conducta (Weinberger, Elvevag y Giedd, 2005), no culminan su desarrollo hasta una vez terminada la pubertad. Por otro lado, el circuito mesolímbico, el encargado de la búsqueda de recompensas, se encuentra influido por alteraciones hormonales propias de esta etapa. Debido a esto, durante los primeros años de la adolescencia se produce un desequilibro entre ambos circuitos cerebrales, fenómeno que puede producir un aumento de búsqueda de sensaciones nuevas de una forma más impulsiva, así como la realización de conductas de riesgo.

Los estilos de vida aprendidos durante este periodo son importantes; ya que, según Telama et al. (2014), los patrones de conducta adquiridos a una edad temprana son aquellos que tienen más probabilidad de ser mantenidos en la adultez, siendo así la adolescencia un periodo clave para la adopción de un estilo de vida u otro.

Durante esta etapa, los jóvenes empiezan a salir sin sus progenitores y el grupo de iguales pasa a tener un papel muy importante en la construcción de la identidad. En este contexto, los jóvenes encuentran un mayor acceso a diferentes sustancias adictivas como lo son el tabaco, el alcohol y el cánnabis (Pérez et al., 2017) Estas y otras conductas de riesgo están influenciadas por el género. Así, Becoña (2002) resalta que el género es un factor significativo en diversas variables, como la disponibilidad de droga, la presión de iguales, el abuso de sustancias en la familia, el estatus socioeconómico y la aculturación.

En la Evaluación del progreso de control de drogas (Organización de los Estados Americanos, 2010) se muestran los siguientes datos de consumo en el año 2008 en Ecuador en 
jóvenes entre 12 y 17 años: el 57\% de chicos y el 37\% de chicas habían consumido alguna vez en la vida tabaco; y el $81 \%$ de chicos y $78.1 \%$ de chicas habían consumido alguna vez en su vida alcohol. En esta encuesta son los chicos los que presentan mayores consumos que las chicas. Chen y Jacobson (2012) concretan que las chicas consumen más que los chicos en la primera adolescencia; mientras que los chicos muestran niveles más altos desde la mitad de la adolescencia hasta principio de la edad adulta. Muñoz y Alvarado (2014) destacan que también puede existir la carga cultural, ya que en Ecuador está mejor visto el consumo en chicos que en chicas.

Por otro lado, la adolescencia es el momento en el que la mayoría de jóvenes tienen sus primeros encuentros sexuales $y$, además, hay una mayor frecuencia de conductas sexuales de riesgo debido a la falta de uso de métodos de barrera. Esta práctica aumenta el riesgo de embarazos no deseados y enfermedades de transmisión sexual (Saeteros, Pérez y Sanabria, 2015). En un estudio de Gonçalves, Castellá y Carlotto (2007), los chicos presentaron mayor número de este tipo de conductas que las chicas y un mayor número de parejas sexuales. Destacan que las conductas sexuales de riesgo tienen una fuerte dimensión social en el desempeño de roles de género. Esto se debe a que mientras que para las chicas es una conducta que está mal vista, a los chicos se les refuerza (Taquette, Vihen y Campos de Paula, 2004).

En referencia a las conductas sexuales de riesgo en Ecuador, según el INEC (2016), datos del 2016 muestran una tasa de 2.6 embarazos en cada 1000 chicas de entre 10 a 14 años y una tasa de 67.8 embarazos en cada 1000 chicas de 15 a 19 años.

Chavez, Chica y Peñaranda (2014) destacan que la población ecuatoriana se inicia tempranamente en las prácticas sexuales y que una cuarta parte no usa métodos de barrera ni otros anticonceptivos. Según estos autores, esto último se relaciona con una baja condición socio-económica así como la escasa información. Este estudio encontró que la mitad de su muestra no tenía conocimientos acertados sobre el tema. Añade Guillén (2015) que Ecuador es uno de los países con menor índice de información sobre anticonceptivos.

Por otro lado, Barahona, Jayo y Puertas (2015), en un estudio cualitativo con una muestra de varones ecuatorianos de entre 15 y 17 años que habían sido padres, destacaron que, aunque la mayoría de los embarazos no habían sido deseados, ellos manifestaban haberlo aceptado desde un principio y asumir esa responsabilidad. Además, Calvo y Varas (2007) destacan que, debido al plano cultural de Ecuador, los embarazos adolescentes son más aceptados por el núcleo familiar que en otros países donde la situación, sobre todo en la familia de la madre adolescente, se vive con más tensión e inestabilidad.

Además, existe una relación entre el consumo de alcohol y el haber mantenido relaciones sexuales en la adolescencia (Ortiz-Melgar, Pérez-Saaedra, Valentín-Ballarta y Zúñiga-Hurtado, 2016). El hecho de conocer las consecuencias negativas de una conducta no evita que se efectúe, sobre todo en adolescentes, ya que se ve reforzada por la emoción experimentada tras realizarla (Sánchez y Muñoz, 2005). Y bajo los efectos del alcohol es más probable que un adolescente tenga una conducta sexual de riesgo sin pensar en las consecuencias (Martínez, Maya, Parada y Duarte, 2013).

Un constructo de la personalidad que se ha visto relacionado con conductas de riesgo es la búsqueda de sensaciones (Malmberg et al., 2013) 
La búsqueda de sensaciones ha sido concretada por Zuckerman (1994) como 'un rasgo definido por la búsqueda de sensaciones y emociones variadas, nuevas, complejas e intensas, y la disposición a asumir riesgos físicos, sociales, legales y financieros con el fin de obtener tales experiencias y sensaciones'. Respecto a las diferencias de género, se han encontrado resultados significativos para todos los niveles de edad, siendo los hombres quienes puntuaban más alto en la búsqueda de sensaciones frente a las mujeres (González-Iglesias, GómezFraguela, Gras y Planes, 2014).

En la revisión realizada en torno a la búsqueda de sensaciones se infiere que éste no es un constructo unitario, sino que incluye varios factores. En este sentido, utilizando la que probablemente es la escala más utilizada para medir el constructo, la Sensation-Seeking Scale$V$, Zuckerman (1994) encuentra que este constructo está formado por cuatro dimensiones. La búsqueda de emociones hace referencia al deseo de implicarse en actividades de riesgo. La búsqueda de experiencias es definida como el deseo de encontrar experiencias nuevas a través de estilos de vida poco convencionales. La desinhibición se refiere a la búsqueda de sensaciones a través de actividades sociales, sexuales o de diversión. Y la susceptibilidad al aburrimiento abarca la baja tolerancia ante las actividades rutinarias o repetitivas (RamosBarbero y Pérez de Albéniz, 2008).

El estudio de Martínez-Lorca y Alonso-Sanz (2003), analizó la relación de esta característica de la personalidad con los diferentes consumos de drogas. Los resultados mostraron que aquellos que puntuaron alto en búsqueda de sensaciones tenían más probabilidad de no rechazar una oferta de tabaco, alcohol y cánnabis; tenían una visión más positiva de estas drogas y habían consumido con más frecuencia. Es decir, parece que la variable de búsqueda de sensaciones juega un papel importante a la hora de predecir la conducta de riesgo de consumo. Estos datos fueron apoyados por otras investigaciones como el estudio de Latorre, Cámara y García (2014) con una muestra de adolescentes entre 12 y 16 años.

Además, en una investigación de Donohew, Zimmerman, Cupp, Novak, Colon y Abell (2000), también se encontró una relación entre la búsqueda de sensaciones y las conductas sexuales de riesgo en una muestra de adolescentes de 15 y 16 años.

Tras la literatura revisada, el objetivo del presente estudio fue conocer, diferenciando por género, qué dimensiones de la búsqueda de sensaciones tienen mayor influencia en las conductas de consumo de drogas y en las conductas sexuales de riesgo en una muestra de adolescentes de Ecuador, así como si este consumo tiene relación con dichas conductas sexuales. Se han planteado las siguientes hipótesis:

Hipótesis 1: Se encontrarán diferencias en función del género en las diferentes conductas de riesgo, siendo los chicos los que realizarán más conductas de riesgo.

Hipótesis 2: La búsqueda de emociones, la búsqueda de experiencias, la desinhibición y la susceptibilidad al aburrimiento actuarán como predictor positivo de las conductas de consumo de alcohol, tabaco y las borracheras tanto en chicos como en chicas.

Hipótesis 3: La búsqueda de emociones, la búsqueda de experiencias, la desinhibición y la susceptibilidad al aburrimiento actuarán como predictor positivo de la conducta sexual de riesgo tanto en chicos como en chicas. 
Hipótesis 4: Las conductas de consumo de alcohol, tabaco y las borracheras actuarán como predictor positivo de la conducta sexual de riesgo tanto en chicos como en chicas.

\section{Método}

\section{Diseño del estudio}

Se diseñó un estudio transversal descriptivo, realizado en un contexto natural en centros educativos en Ecuador en menores de entre 13 y 16 años.

\section{Participantes}

En este estudio se ha contado con una muestra de 367 participantes procedentes de diferentes centros educativos de Ecuador. En total la muestra se compone de 204 chicos $(55.6 \%)$ y 163 chicas (44.4\%), todos ellos con edades comprendidas entre 13 y 16 años, siendo la media de 14.42 años $(\mathrm{DE}=1.08)$. Un $26.4 \%$ de la muestra tiene 13 años, un $24.8 \%$ tiene 14 años, un $29.3 \%$ tiene 15 años y un $19.6 \%$ tiene 16 años.

\section{Instrumentos}

Datos sociodemográficos. Para conocer las características sociodemográficas, se realizaron preguntas relacionadas con el género, la edad y la fecha de nacimiento.

Conductas de riesgo. Para conocer las conductas de riesgo, se utilizaron algunos ítems del cuestionario The Adolescent Risk-Taking Questionnaire traducido al castellano (Gullone, Moore, Moss y Boyd, 2000), concretamente, del cuestionario de conductas de riesgo. Estos fueron el ítem 1 para medir la conducta de consumo de tabaco, el ítem 8 para medir la conducta de consumo de alcohol, el ítem 14 para medir la conducta de borrachera y el ítem 16 para medir la conducta sexual de riesgo. Los sujetos responden a una escala tipo Likert de 5 puntos: nunca lo he hecho (0), casi nunca lo hago (1), lo hago a veces (2), lo hago bastante (3) y lo hago mucho (4).

Búsqueda de sensaciones. Para medir el rasgo 'Búsqueda de sensaciones' se ha utilizado la versión en castellano de la escala Sensation-Seeking Scale-V (Zuckerman, Eysenck y Eysenck, 1978; Pérez y Torrubia, 1986). Consta de 40 ítems que el sujeto ha de contestar afirmativa o negativamente. La escala se compone de cuatro subescalas de 10 ítems cada una. Estas cuatro subescalas son: Búsqueda de Emociones (BEM), Búsqueda Experiencias (BEX), Desinhibición (DES) y Susceptibilidad hacia el Aburrimiento (SAB). El Alfa de Cronbach en todas las escalas está entre el .52 y el .78.

\section{Procedimiento}

La muestra se seleccionó a través de un muestreo no probabilísitico de tipo intencional durante una estancia de una de las investigadoras (G. L-C.) en la ciudad de Machala en el año 2014. Para lograr la mayor representatividad posible, los criterios de inclusión fueron ser estudiantes de centros de educación, tanto públicos como privados de la ciudad, y tener una edad comprendida entre los 13 y los 17 años. En concreto, se logró la colaboración de dos centros privados y dos públicos.

Tras explicar el objetivo y procedimiento del estudio, se obtuvo el consentimiento por parte de la dirección de los diferentes colegios para llevar a cabo la investigación. Con la cola- 
boración del colegio se seleccionaron diferentes grupos de clase en la que recoger la información. Concretamente, en cada colegio se seleccionaron tres grupos de diferentes edades. A cada grupo se le explicaba el objetivo del estudio, incidiendo en la voluntariedad de la participación y la confidencialidad de los datos. A continuación, se suministraban los cuestionarios (ver apartado instrumentos) en papel, grapados para asegurar que todos los alumnos los completaran en el mismo orden. Los investigadores permanecían en el aula hasta que finalizaba la recogida de datos, resolviendo las dudas que surgían entre los alumnos.

\section{Análisis de datos}

Para la realización de los análisis estadísticos convenientes, se utilizó el programa estadístico SPSS Statics 23. En primer lugar, se segregó la muestra respecto al género para obtener los siguientes análisis en chicos y en chicas. En segundo lugar, se realizó un análisis de fiabilidad para conocer el Alfa de Cronbach de cada una de las subescalas de la escala Sensation-Seeking Scale-V. En tercer lugar, se realizaron los descriptivos pertinentes para conocer las frecuencias de las conductas de riesgo. En cuarto lugar, se realizaron correlaciones simples para conocer la interacción entre las diferentes variables. Y por último se realizaron regresiones simples para conocer el peso de cada variable dependiente en la independiente y así conocer los resultados de las hipótesis planteadas.

\section{Resultados}

\section{Descriptivos}

Los estadísticos descriptivos referentes a la frecuencia de las conductas de riesgo estudiadas segregados por género son los siguientes:

Los chicos, respecto a la conducta de consumo de tabaco, presentan los siguientes datos: el $67.7 \%$ nunca lo ha consumido, el $16.2 \%$ casi nunca, el $11.8 \%$ a veces, el $2.9 \%$ bastantes veces y el $1.5 \%$ muchas veces. Respecto a la conducta de consumo de alcohol: el $63.7 \%$ nunca lo ha consumido, el $14.2 \%$ casi nunca, el $16.7 \%$ a veces, el $2.9 \%$ bastantes veces y el $2.5 \%$ muchas veces. En la conducta de borrachera se encuentra lo siguiente: el $70.6 \%$ nunca se ha emborrachado, el $13.7 \%$ casi nunca, el $12.7 \%$ a veces, el $1.5 \%$ bastantes veces y el $1.5 \%$ muchas veces. Y respecto a la conducta de sexual de riesgo: el $72.6 \%$ nunca las ha realizado, el $13.7 \%$ casi nunca, el $11.3 \%$ a veces, el $0.5 \%$ bastantes veces y el $2 \%$ muchas veces.

Las chicas, respecto a la conducta de consumo de tabaco, presentan las siguientes frecuencias: el $87.7 \%$ nunca lo ha consumido, el $7.4 \%$ casi nunca, el $4.3 \%$ a veces y el $.6 \%$ bastantes veces. Respecto a la conducta de consumo de alcohol: el $77.3 \%$ nunca lo ha consumido, el $10.4 \%$ casi nunca, el $9.8 \%$ a veces, el .6\% bastantes veces y el $1.8 \%$ muchas veces. En la conducta de borrachera se encuentra que: el $85.9 \%$ nunca se ha emborrachado, el $7.4 \%$ casi nunca, el $4.9 \%$ a veces, el $0.6 \%$ bastantes veces y el $1.2 \%$ muchas veces. Y en referencia a la conducta de sexual de riesgo: el $96.3 \%$ nunca las ha realizado, el $1.2 \%$ casi nunca y el $2.5 \%$ a veces.

Respecto al Alfa de Cronhbach de las subescalas de la escala de búsqueda de sensaciones, las puntuaciones encontradas fueron las siguientes: para la búsqueda de emociones se ob- 
tuvo una puntuación de .74, para la búsqueda de experiencias se obtuvo una puntuación de .43, para la desinhibición, una puntuación de .76; y para la susceptibilidad al aburrimiento, una puntuación de .55 .

Diferencias en las conductas de riesgo según el género del alumnado

Se realizó un análisis de diferencia de medias de las variables dependientes (ANOVA) teniendo como factor el género de la muestra. Tal y como muestra la Tabla 3, se encontraron diferencias en todas las conductas de riesgo. En relación a estas diferencias, los chicos presentaron mayor consumo de tabaco $(\mathrm{F}=20.68, \mathrm{p}=.00)$, alcohol $(\mathrm{F}=7.18, \mathrm{p}=.00)$, mayor frecuencia de borracheras $(\mathrm{F}=9.48, \mathrm{p}=.00)$ y mayor frecuencia de conductas sexuales de riesgo $(\mathrm{F}=30.60, \mathrm{p}=.00)$.

Figura 1. Resultados diferenciados según el género del alumnado

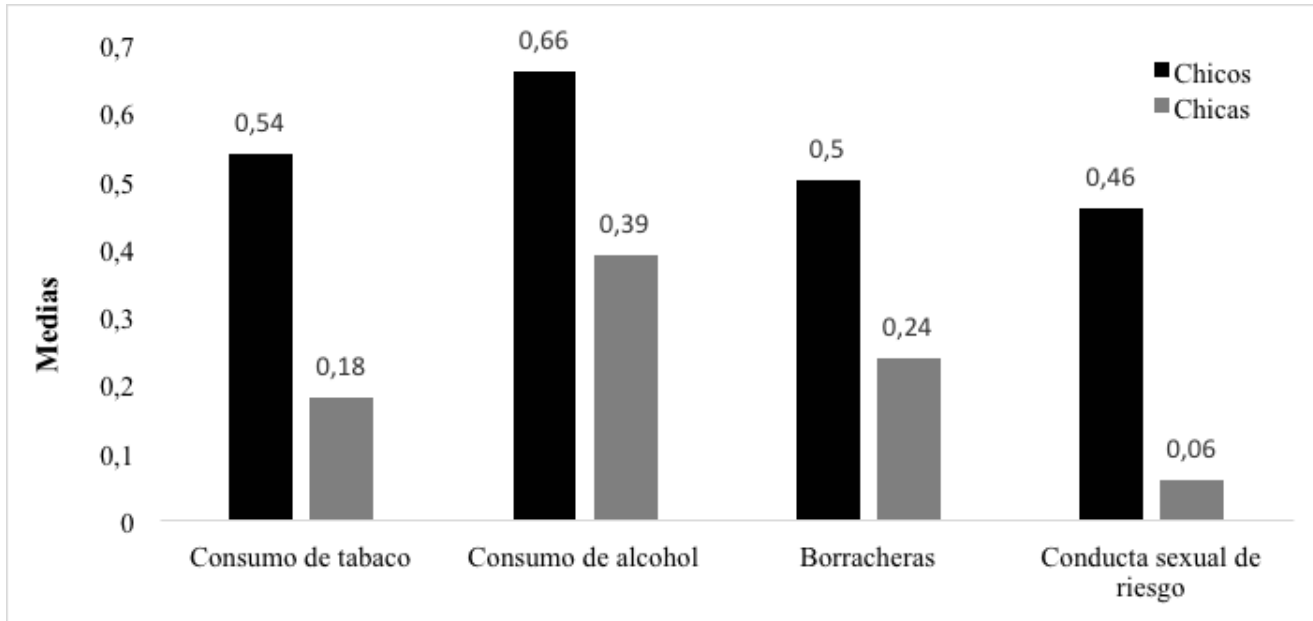

Conductas de riesgo

Análisis de regresión lineal simple de la búsqueda de emociones, búsqueda de experiencias, desinhibición y susceptibilidad al aburrimiento sobre las conductas de consumo de tabaco

Se realizaron cuatro análisis de regresión lineal simple para estudiar si la búsqueda de emociones, la búsqueda de experiencias, la desinhibición y la susceptibilidad al aburrimiento actúan como predictores positivos de la conducta de consumo de tabaco (tabla 1).

La Tabla 1 muestra los resultados de los análisis de regresión simple de las diferentes dimensiones de la búsqueda de sensaciones sobre el consumo de tabaco. Como puede observarse, tanto la búsqueda de experiencias como la desinhibición predicen el consumo de tabaco tanto en chicas como en chicos. En concreto, la búsqueda de experiencias explica el 11 y $5 \%$ de la varianza en chicos y chicas, respectivamente, mientras que la desinhibición 
Tabla 1. Análisis de regresión lineal simple de la búsqueda de emociones, búsqueda de experiencias, desinhibición y susceptibilidad al aburrimiento sobre las conductas de consumo de tabaco.

\begin{tabular}{lccccccccc}
\hline Variable & \multicolumn{2}{c}{$\mathrm{R}^{2}$} & \multicolumn{2}{c}{ Beta } & \multicolumn{1}{c}{$\mathrm{t}$} & \multicolumn{3}{c}{ Sig. } \\
\hline & $\mathrm{H}$ & $\mathrm{M}$ & $\mathrm{H}$ & $\mathrm{M}$ & $\mathrm{H}$ & $\mathrm{M}$ & $\mathrm{H}$ & $\mathrm{M}$ \\
Tabaco & .06 & .00 & & & & & & \\
- Búsqueda de emociones & & & .06 & -.00 & .80 & -.01 & .42 & .99 \\
$\begin{array}{l}\text { Tabaco } \\
\text { - Búsqueda de experiencias }\end{array}$ & $.11^{* *}$ & $.05^{* *}$ & & & & & & \\
Tabaco & & & .33 & .22 & 5.04 & 2.90 & .00 & .00 \\
- Desinhibición & $.22^{* *}$ & $.14^{* *}$ & & & & & & \\
Tabaco & & & .47 & .37 & 7.65 & 5.04 & .00 & .00 \\
- Susceptibilidad al Aburrimiento & $.02^{*}$ & .01 & & & & & & \\
\hline
\end{tabular}

Nota: $* \mathrm{p}<.05, * * \mathrm{p}<.01 ; \mathrm{H}=$ Hombre, $\mathrm{M}=$ Mujer

explica el 22 y $14 \%$ de la varianza, respectivamente (todas las $\mathrm{p}<.01$ ). La susceptibilidad al aburrimiento explica el $2 \%$ de la varianza en chicos $(\mathrm{p}<.05)$.

Análisis de regresión simple de la búsqueda de emociones, búsqueda de experiencias, desinhibición y la susceptibilidad al aburrimiento sobre las conductas de consumo de alcohol

Tabla 2. Análisis de regresión simple de la búsqueda de emociones, búsqueda de experiencias, desinhibición y la susceptibilidad al aburrimiento sobre las conductas de consumo de alcohol

\begin{tabular}{|c|c|c|c|c|c|c|c|c|}
\hline \multirow[t]{2}{*}{ Variable } & \multicolumn{2}{|c|}{$\mathrm{R}^{2}$} & \multicolumn{2}{|c|}{ Beta } & \multicolumn{2}{|c|}{$\mathrm{t}$} & \multicolumn{2}{|c|}{ Sig. } \\
\hline & $\mathrm{H}$ & $\mathrm{M}$ & $\mathrm{H}$ & $\mathrm{M}$ & $\mathrm{H}$ & $M$ & $\mathrm{H}$ & $\mathrm{M}$ \\
\hline Alcohol & .00 & .02 & & & & & & \\
\hline - Búsqueda de emociones & & & .05 & .13 & .69 & 1.63 & .49 & .11 \\
\hline Alcohol & $.08^{* *}$ & $.10^{* *}$ & & & & & & \\
\hline - Búsqueda de experiencias & & & .29 & .32 & 4.30 & 4.26 & .00 & .00 \\
\hline Alcohol & $.24 * *$ & $.15^{* *}$ & & & & & & \\
\hline - Desinhibición & & & .49 & .39 & 7.92 & 5.42 & .00 & .00 \\
\hline Alcohol & .01 & .02 & & & & & & \\
\hline $\begin{array}{l}\text { - Susceptibilidad al aburri- } \\
\text { miento }\end{array}$ & & & .09 & .14 & 1.24 & 1.79 & .22 & .08 \\
\hline
\end{tabular}

Nota: $* \mathrm{p}<.05, * * \mathrm{p}<.01 ; \mathrm{H}=$ Hombre, $\mathrm{M}=$ Mujer 
Se realizaron cuatro análisis de regresión lineal simple para estudiar si la búsqueda de emociones, la búsqueda de experiencias, la desinhibición y la susceptibilidad al aburrimiento actúan como predictores positivos de la conducta de consumo de alcohol (tabla 2).

La Tabla 2 muestra los resultados de los análisis de regresión simple de las diferentes dimensiones de la búsqueda de sensaciones sobre el consumo de alcohol. Como puede observarse, tanto la búsqueda de experiencias como la desinhibición predicen el consumo de alcohol tanto en chicas como en chicos. En concreto, la búsqueda de experiencias explica el 8 y $10 \%$ de la varianza en chicos y chicas, respectivamente, mientras que la desinhibición explica el 24 y $15 \%$ de la varianza, respectivamente (todas las p <.01).

Análisis de regresión simple de la búsqueda de emociones, búsqueda de experiencias, desinhibición y la susceptibilidad al aburrimiento sobre las conductas de borracheras

Se realizaron cuatro análisis de regresión lineal simple para estudiar si la búsqueda de emociones, la búsqueda de experiencias, la desinhibición y la susceptibilidad al aburrimiento actúan como predictores positivos de la conducta de borrachera (Tabla 3).

Tabla 3. Análisis de regresión simple de la búsqueda de emociones, búsqueda de experiencias, desinhibición y la susceptibilidad al aburrimiento sobre las conductas de borracheras

\begin{tabular}{|c|c|c|c|c|c|c|c|c|}
\hline \multirow[t]{2}{*}{ Variable } & \multicolumn{2}{|c|}{$\mathrm{R}^{2}$} & \multicolumn{2}{|c|}{ Beta } & \multicolumn{2}{|c|}{$\mathrm{t}$} & \multicolumn{2}{|c|}{ Sig. } \\
\hline & $\mathrm{H}$ & $\mathrm{M}$ & $\mathrm{H}$ & $\mathrm{M}$ & $\mathrm{H}$ & $\mathrm{M}$ & $\mathrm{H}$ & $\mathrm{M}$ \\
\hline Borracheras & .00 & .01 & & & & & & \\
\hline - Búsqueda de emociones & & & .04 & .10 & .60 & 1.25 & .55 & .2 \\
\hline Borracheras & $.08^{* *}$ & $.11^{* *}$ & & & & & & \\
\hline - Búsqueda de experiencias & & & .28 & .32 & 4.13 & 4.35 & .00 & .0 \\
\hline Borracheras & $.29 * *$ & $.24 * *$ & & & & & & \\
\hline - Desinhibición & & & .54 & .49 & 9.02 & 7.18 & .00 & .0 \\
\hline Borracheras & $.03^{*}$ & .09 & & & & & & \\
\hline - Susceptibilidad al aburrimiento & & & .16 & .14 & 2.25 & 1.74 & .03 & .08 \\
\hline
\end{tabular}

Nota: $* \mathrm{p}<.05, * * \mathrm{p}<.01 ; \mathrm{H}=$ Hombre, $\mathrm{M}=$ Mujer

La Tabla 3 muestra los resultados de los análisis de regresión simple de las diferentes dimensiones de la búsqueda de sensaciones sobre la conducta de borrachera. Como puede observarse, tanto la búsqueda de experiencias como la desinhibición la conducta de borrachera tanto en chicas como en chicos. En concreto, la búsqueda de experiencias explica el 8 y $11 \%$ de la varianza en chicos y chicas, respectivamente, mientras que la desinhibición explica el 29 y $24 \%$ de la varianza, respectivamente (todas las p < .01). La susceptibilidad al aburrimiento explica el $3 \%$ de la varianza en chicos $(\mathrm{p}<.05)$. 
Análisis de regresión simple de la búsqueda de emociones, búsqueda de experiencias, desinhibición y la susceptibilidad al aburrimiento sobre la conducta sexual de riesgo

Se realizaron cuatro análisis de regresión lineal simple para estudiar si la búsqueda de emociones, la búsqueda de experiencias, la desinhibición y la susceptibilidad al aburrimiento actúan como predictores positivos de la conducta sexual de riesgo (tabla 4).

Tabla 4. Análisis de regresión simple de la búsqueda de emociones, búsqueda de experiencias, desinhibición y la susceptibilidad al aburrimiento sobre la conducta sexual de riesgo

\begin{tabular}{|c|c|c|c|c|c|c|c|c|}
\hline \multirow[t]{2}{*}{ Variable } & \multicolumn{2}{|c|}{$\mathrm{R}^{2}$} & \multicolumn{2}{|c|}{ Beta } & \multicolumn{2}{|c|}{$\mathrm{t}$} & \multicolumn{2}{|c|}{ Sig. } \\
\hline & $\mathrm{H}$ & M & $\mathrm{H}$ & M & $\mathrm{H}$ & M & $\mathrm{H}$ & M \\
\hline Conducta sexual de riesgo & .02 & $.04^{*}$ & & & & & & \\
\hline - Búsqueda de emociones & & & .12 & -.19 & 1.74 & -2.48 & .08 & .01 \\
\hline Conducta sexual de riesgo & $.04 * *$ & .01 & & & & & & \\
\hline - Búsqueda de experiencias & & & .21 & -.09 & 3.05 & -1.17 & .00 & .25 \\
\hline Conducta sexual de riesgo & $.16^{* *}$ & .00 & & & & & & \\
\hline - Desinhibición & & & .39 & .06 & 6.09 & .72 & .00 & .47 \\
\hline Conducta sexual de riesgo & .01 & .00 & & & & & & \\
\hline - Susceptibilidad al aburrimiento & & & .12 & -.01 & 1.69 & -.183 & .09 & .86 \\
\hline
\end{tabular}

Nota: $* \mathrm{p}<.05, * * \mathrm{p}<.01 ; \mathrm{H}=$ Hombre, $\mathrm{M}=$ Mujer

La Tabla 4 muestra los resultados de los análisis de regresión simple de las diferentes dimensiones de la búsqueda de sensaciones sobre la conducta sexual de riesgo. Como puede observarse, tanto la búsqueda de experiencias como la desinhibición la conducta de borrachera en chicos. En concreto, la búsqueda de experiencias explica el $4 \%$ de la varianza en chicos, mientras que la desinhibición explica el $16 \%$ de la varianza (todas las $\mathrm{p}<.01$ ). La búsqueda de emociones explica el $4 \%$ de la varianza en chicas $(\mathrm{p}<.05)$.

Análisis de regresión simple las conductas de consumo de tabaco, alcohol y borrachera sobre la conducta sexual de riesgo

Se realizaron cuatro análisis de regresión lineal simple para estudiar si la conducta de consumo de tabaco, alcohol y las borracheras actúan como predictores positivos de la conducta sexual de riesgo (tabla 5).

La Tabla 5 muestra los resultados de los análisis de regresión simple de las diferentes conductas de consumo sobre la conducta sexual de riesgo. Como puede observarse, tanto el consumo de tabaco y alcohol como la conducta borrachera predicen la conducta sexual de riesgo tanto en chicas como en chicos. En concreto, el consumo de tabaco explica el 13 y 9\% de la varianza en chicos y chicas, respectivamente; el consumo de alcohol explica el 12 y $5 \%$ de la varianza en chicos y chicas, respectivamente; y la conducta de borrachera explica el 14 y $10 \%$, respectivamente. 
Tabla 5. Análisis de regresión simple las conductas de consumo de tabaco, alcohol y borrachera sobre la conducta sexual de riesgo

\begin{tabular}{lcccccccc}
\hline Variable & \multicolumn{2}{c}{$\mathrm{R}^{2}$} & \multicolumn{2}{c}{ Beta } & \multicolumn{1}{c}{$\mathrm{t}$} & \multicolumn{2}{c}{ Sig. } \\
\hline & $\mathrm{H}$ & $\mathrm{M}$ & $\mathrm{H}$ & $\mathrm{M}$ & $\mathrm{H}$ & $\mathrm{M}$ & $\mathrm{H}$ & $\mathrm{M}$ \\
Conducta sexual de riesgo & $.13^{* *}$ & $.09^{* *}$ & & & & & & \\
- Tabaco & & & .36 & .30 & 5.40 & 3.91 & .00 & .00 \\
Conducta sexual de riesgo & $.12^{* *}$ & $.05^{* *}$ & & & & & & \\
- Alcohol & & & .35 & .22 & 5.27 & 2.91 & .00 & .00 \\
Conducta sexual de riesgo & $.14^{* *}$ & $.10^{* *}$ & & & & & & \\
- Borrachera & & & .37 & .32 & 5.72 & 4.28 & .00 & .00 \\
\hline
\end{tabular}

Nota: $* \mathrm{p}<.05, * * \mathrm{p}<.01 ; \mathrm{H}=$ Hombre, $\mathrm{M}=$ Mujer

\section{Discusión}

El objetivo de esta investigación era conocer si la búsqueda de sensaciones y sus diferentes dimensiones están implicadas en las conductas de consumo de drogas y en las conductas sexuales de riesgo, y si estas dos conductas de riesgo están a su vez relacionadas entre sí, examinando además el papel del género en dichas relaciones.

Los resultados muestran que, tanto en chicos como chicas, la conducta de riesgo más realizada es el consumo de alcohol. Esto se explica por la normalización del consumo en la sociedad actual. Castaño, Arango, Morales, Rodríguez y Montoya (2013) apuntan que, aunque los jóvenes tienden al policonsumo, el alcohol sigue siendo la sustancia de mayor consumo.

La primera hipótesis planteaba que se encontrarían diferencias en función del género en las diferentes conductas de riesgo, siendo los chicos los que realizarán más conductas de riesgo. Según el ANOVA las diferencias entre géneros son significativas en todas las conductas de riesgo. Son los chicos aquellos que presentan una mayor frecuencia en consumo de tabaco, alcohol y borracheras apoyando así los resultados de otros estudios (Khan, Cleland, Scheidell y Berger, 2014). Llama especialmente la atención que el 3.7\% de chicas ha realizado conductas sexuales de riesgo, mientras que este porcentaje en chicos es el $27.4 \%$, coincidiendo estos resultados con la literatura (Gonçalves, Castellá y Carlotto, 2007)

La segunda hipótesis planteaba que la búsqueda de emociones, la búsqueda de experiencias, la desinhibición y la susceptibilidad al aburrimiento actuaban como predictor positivo de las conductas de consumo de tabaco, alcohol y las borracheras tanto en chicos como en chicas. Según el análisis de regresión lineal simple, la búsqueda de experiencias y la desinhibición explican, tanto en chicos como en chicas, parte del consumo de tabaco, alcohol y de la conducta de borrachera. Sin embargo, la susceptibilidad al aburrimiento sólo explica parte del consumo de tabaco en chicos. Por lo tanto, podríamos decir que la hipótesis se mantiene parcialmente. Otros estudios como el de Inglés et al. (2007), han encontrado que la búsqueda de sensaciones es el mejor predictor del consumo de tabaco y alcohol en adolescentes. 
Nuestro estudio va un paso más allá, mostrando que no todas las dimensiones del constructo predicen en la misma medida la realización de conductas de riesgo, y constatando que estas dimensiones predicen en mayor medida las conductas de riesgo realizadas por los chicos que por las chicas.

La tercera hipótesis planteaba que la búsqueda de emociones, la búsqueda de experiencias, la desinhibición y la susceptibilidad al aburrimiento actuarán como predictor positivo de la conducta sexual de riesgo chicos como en chicas. Según el análisis de regresión lineal simple, la búsqueda de experiencias y la desinhibición explican la conducta sexual de riesgo sólo en chicos rechazándose así la hipótesis. Esto se puede deber a que las chicas están más concienciadas respecto a los riesgos que conlleva no usar preservativo, mientras que más chicos lo ven como una barrera (Giménez-García, Ballester-Arnal, Gil-Llario, CárdenadLópez y Duran-Baca, 2012).

Y la cuarta hipótesis planteaba que las conductas de consumo de alcohol, tabaco y las borracheras actuarán como predictor positivo de la conducta sexual de riesgo tanto en chicos como en chicas. Según el análisis de regresión lineal simple, los dos consumos y las borracheras explicaban la conducta sexual de riesgo tanto en chicos como en chicas. La hipótesis se mantiene. Según Castaño et al. (2013), es el alcohol la sustancia que más influencia tiene dentro de la conducta sexual de riesgo pero que también se ve relacionada con otras como la marihuana, la cocaína o el éxtasis. Bellis et al. (2008), encontraron que aquellos jóvenes que se habían emborrachado alguna vez en el último mes, reportaban mayor número de parejas sexuales sin haber usado preservativo. Se observa que el género es una variable importante a tener en cuenta, ya que se predice un mayor porcentaje de la varianza en chicos que en chicas.

En conclusión, es mucho más probable que aquellos jóvenes que puntúan alto en la variable de búsqueda de sensaciones, se inicien en el consumo de estas drogas o realicen conductas sexuales de riesgo. Es necesario identificar a los sujetos en situación de riesgo precozmente para canalizar esas ganas de experiencias nuevas hacia otras conductas saludables como el deporte de aventura. De hecho, González, de la Puente, Rodríguez y González (2000) encontraron que tanto personas consumidoras de drogas como personas que realizaban habitualmente deportes de riesgo, puntuaban alto en la escala de búsqueda de sensaciones. Resultados muy similares presentó el estudio deGil-Olarte, Cavalcante, Paramio, Zayaz y Guil (2017). Se evidencia la necesidad de incrementar la oferta de programas sobre educación sexual en los centros educativos de este país para trabajar tanto en la prevención de embarazos no deseados como en la prevención del contagio de enfermedades de transmisión sexual, poniendo especial interés en los chicos.

De hecho, en el Plan Nacional de Desarrollo 2017-2021 “Toda una vida" que se está llevando a cabo en Ecuador, en el objetivo "Derechos para todos durante toda la vida" se recoge el siguiente punto "es prioritaria la ampliación de la cobertura de salud traducida en programas, proyectos y atención a problemas emergentes tales como el consumo de drogas, el alcoholismo, el embarazo precoz, la violencia intrafamiliar, la violencia sexual y demás problemas psicosociales que afectan a los niños, niñas y adolescentes". Por lo tanto, son 
necesarias las investigaciones en esta línea en Ecuador para, gracias a la evidencia, cumplir con dichos propósitos.

En referencia a las limitaciones del estudio, por un lado, las respectivas a la muestra, ya que el tamaño es pequeño y procede de una única zona. Sería interesante conocer los resultados en otros lugares geográficos, así como con una mayor muestra. También se trata de un estudio trasversal, lo que impide que se hagan relaciones causales entre las variables de interés. Para otras líneas de investigación sería interesante conocer si existen diferencias según la etapa de la adolescencia o trabajar con otras variables de personalidad como la impulsividad.

\section{Referencias}

Barahona, P., Jayo, L., y Puertas, R. (2015). Percepción de los adolescentes varones sobre paternidad adolescente. Revista PUCE, 100, 33-54.

Becoña, E. (2000). Los adolescentes y el consume de drogas. Papeles del Psicólogo, 77, 25-32.

Bellis, M., Hughes, K., Calafat, A., Juan, M., Ramon, A., Rodríguez, J., y Phillips-Howard, P. (2008). Sexual uses of alcohol and drugs and the associated health risks: a cross sectional study of young people in nine European cities. MC public health, 8 (1), 155.

Calvo, P. y Varas, J. (2007). Entorno psicosocial en madres adolescentes. Revista Obstetricia y Ginecología, 2 (2), 178-180.

Castaño, G., Arango, E., Morales, S., Rodríguez, A. y Montoya, C. (2013). Riesgos y consecuencias de las prácticas sexuales en adolescentes bajo los efectos de alcohol y otras drogas. Revista Cubana de Pediatría, 85 (1), 36-50.

Chavez, J. A., Chica, P. A., y Peñaranda, R. I. (2014). Determinantes del comportamiento sexual y reproductivo de las y los adolescentes en colegios urbanos y rurales de la Ciudad de Macas. Universidad de Cuenca, Ecuador.

Chen, P., y Jacobson, K. C. (2012). Developmental trajectories of substance use from early adolescence to young adulthood: Gender and racial/ethnic differences. Journal of adolescent health, 50 (2), 154-163.

Gil-Olarte, P., Cavalcante, A. C., Paramio, A., Zayas, A. y Guil, R. (2017). Impulsividad y búsqueda de sensaciones: implicaciones de intervención en jóvenes. Revista INFAD de Psicología, 2 (1), 393-404.

Giménez-García, C., Ruiz-Palomino, E., Gil-Llario, M. D., Ballester-Arnal, R., y Castro-Calvo, J. (2016). Una perspectiva de género en el estudio de las conductas de riesgo de los adolescentes. International Journal of Developmental and Educational Psychologu, 1 (2), 189-198.

Gonçalves, S., Castellá, J. y Carlotto. M. S. (2007). Predictores de Conductas Sexuales de Riesgo entre Adolescentes. Revista Interamericana de Psicología, 41(2), 161-166.

González, JL., De la Puente, E., Rodríguez, JM. y González, I. (2000). La prevención de drogodependencias mediante actividades cooperativas de riesgo y aventura. Apuntes: Educación física y deportes, 59, 46-54.

González-Iglesias, B., Gómez-Fraguela, J.A., Gras, M.E. y Planes, M. (2014). Búsqueda de sensaciones y consumo de alcohol: El papel mediador de la percepción de riesgos y beneficios. Anales de Psicología, 30 (3), 1061-1068.

Guillén, A. M. (2015). Nivel de conocimiento y percepción de los y las adolescentes de 14 a 18 años sobre el uso de métodos anticonceptivos frente a la prevención del embarazo no deseado en la institución educativa ludoteca de septiembre a diciembre del 2014. Universidad Central de Ecuador: Quito.

Gullone, E., Moore, S., Moss, S., y Boyd, C. (2000). The adolescent risk-taking questionnaire: Development and psychometric evaluation. Journal of Adolescent Research, 15 (2), 231-250. 
Inglés, C., Delgado, B., Bautista, R., Torregrosa, M., Espada, J., García-Fernández, J., Hidalgo, M. y García-López, L. (2007). Factores psicosociales relacionados con el consumo de alcohol y tabaco en adolescentes españoles. Internacional Journal of Clinical and Health Psychology, 7 (2), 403-420.

Instituto nacional de estadística y censos. (2016). Estadísticas Vitales: Registro estadístico de nacidos vivos y defunciones. Gobiernos Nacional de la República de Ecuador: Quito.

Khan, M. R., Cleland, C. M., Scheidell, J. D. y Berger, A. T. (2014). Gender and racial/ethnic differences in patterns of adolescent alcohol use and asociations with adolescent and adult illicit drug use. The American journal of drug and alcohol abuse, 40 (3), 213-224

Latorre, P. A., Cámara, J. C. y García, F. (2014). Búsqueda de sensaciones y hábitos de tabaquismo, consumo de alcohol y práctica deportiva en estudiantes de Educación Secundaria. Salud mental, 37 (2), 145-152.

Malmberg, M., Kleinjan, M., Overbeek, G., Vermulst, A. A., Lammers, J. y Engels, R. C. (2013). Are there reciprocal relationships between substance use risk personality profiles and alcohol or tobacco use in early adolescence? Addictive Behaviors, 38, 2851-2859.

Martínez-Lorca, M. y Alonso-Sanz, C. (2003). Búsqueda de sensaciones, autoconcepto, asertividad y consumo de drogas ¿Existe relación? Adicciones, 15 (2), 145-158.

Martínez, J., Maya, N., Parada, L. y Duarte, K. (2013). Diferencias de las prácticas sexuales entre hombres y mujeres en estudiantes universitarios durante el primer semestre. Revista Duazary, 10 (2), 112-118.

Matthys, W., Vanderschuren, L. J. y Schutter, D.J. (2013). The neurobiology of oppositional defiant disorder and conduct disorder: Altered functioning in three mental domains. Development and Psychopathology, 25, 193-207.

Muñoz, S. L. y Alvarado, R. (2014). Perfil socio-demográfico y psicosocial de las adolescentes embarazadas en una maternidad de Guayaquil, Ecuador. Universidad de Chile: Santiago.

Olivia, A. (2007). Desarrollo cerebral y asunción de riesgos durante la adolescencia. Apuntes de Psicología, 25 (3)، 239-254.

Organización de los Estados Americanos. (2010). Mecanismo de Evaluación Multilateral: Evaluación del progreso de control de drogas 2007-2009 en Ecuador. Washington: CICAD.

Organización Mundial de la Salud. (1998). Promoción de la salud: Glosario. Ginebra: World Health Organization.

Ortiz- Melgar, M., Pérez-Saavedra, V., Valentín-Ballarta, J. y Zúñiga- Hurtado, A. (2015). Asociación entre consumo de alcohol y relaciones sexuales ocasionales en los adolescentes. Revista enfermería Herediana, 8 (2), 109-114.

Pérez, A., Redondo, M., Martínez, M. L., Jiménez, I., Mesa, I., y Leal, F. J. (2017). Cambios en el consumo alcohólico de riesgo en población adolescente en la última década (2004-2013): Una aproximación cuanti-cualitativa. Atención Primaria, 49 (9), 525-533.

Pineda, S. y Aliño, M. (2002) El concepto de adolescencia. En R. Márquez y E. F. Colás, Manual de prácticas clínicas para la atención integral a la salud en la adolescencia, 2 ed. (pp. 15-23). Ciudad de la Habana: Ministerio de Salud Pública.

Ramos Barbero, V. y Pérez de Albéniz, G. (2008). La variable de personalidad búsqueda de sensaciones como factor de riesgo en el consumo de drogas. International Journal of Developmental and Educational Psychology, 1 (4), 169-178.

Saeteros, R. C., Pérez, J. y Sanabria, G. (2015) Conducta de riesgo y problemas sexuales y reproductivos de estudiantes universitarios ecuatorianos. Humanidades méicas, 15 (3), 421-439.

Sánchez, M. y Muñoz, A. (2005). Influencia de padres y amigos sobre la actitud hacia las conductas sexuales de prevención en la adolescencia. Un análisis en función del género. Revista Latinoamericana de Psicología, 37, 71-79.

Senplades. (2017). Plan Nacional de Desarrollo 2017-2021: Toda una vida. Senplades: Quito.

Taquette, S. R., Vilhena, M. M., y Campos de Paula, M. (2004). Doenças sexualmente transmissíveis e gênero: um estudo transversal com adolescentes no Rio de Janeiro. Cadernos de Saúde Pública, 20 (1), 282-290.

Telama, R., Yang, X., Leskinen, E., Kankaanpaa, A., Hirvensalo, M., Tammelin, T.,...Raitakari, 0.T. (2014). Tracking of physi- 
cal activity from early childhood through youth into adulthood. Medicine \& Science in Sports \& Exercise, 46, 955-962.

Toro-Trallero, J. (2010). El adolescente en su mundo. Riesgos, problemas y trastornos. Madrid: Pirámide.

Weinberger, D. R., Elvevag, B. y Giedd, J.N. (2005). The adolescent brain: A work in progress. Washington, DC: National Campaign to Prevent Teen Pregnancy.

Zuckerman, M., Eysenck, S. y Eysenck, H.J. (1978). Sensation seeking in England and America: Crosscultural age and sex comparisons. Journal of Consulting and Clinical Psychology, 46, 139-149.

Zuckerman, M. (1994). Behavioral expressions and biosocial bases of sensation seeking. Cambridge university press. 